\title{
On Load Single Phase Solid State Tap Changer
}

\author{
Mohammad H. Taha \\ Electrical and Computer Engineering Department, Rafik Hariri University, Lebanon \\ (TahaMH@rhu.edu.lb)
}

Corresponding Author; Mohammad H. Taha, Electrical and Computer Engineering Department, Rafik Hariri University, Lebanon, Tel: +9615603090, Fax: +9615601380, TahaMH@ rhu.edu.lb

Received: 04.11.2016 Accepted: 01.06.2016

\begin{abstract}
In electric energy transmission and distribution system, voltage control is an essential part to maintain proper voltage limit at the consumer's terminal. On-load tap-changers are indispensable in regulating power transformers used in electrical energy networks and industrial applications. General switching principles and application for the On-load tap-changers are discussed and presented. A single phase Tap-Changer using a GTO with antiparallel thyristor to perform switching of one upward or downward transition is described in this paper. The logic of operation, simulation and experimental results for resistive, inductive loads are presented.
\end{abstract}

Keywords: Voltage control, thyristors, tap-changer, loads.

\section{Introduction}

For more than 100 years on load tap changer was the essential part in general electrical power installation. The main task of on load tap changer is the ability to regulate output voltage without any interruption on any electric network. This can be done by monitoring the output voltage and changing the turns ration between the primary and secondary winding in order to change the level of the secondary voltage. [1-3].

On-load Tap-Changers are used when load disconnection is not acceptable, so its main task is to transfer the transformer load current from one regulating winding tapping to another without any interruption of the current from the transformer to the load. However, on load tap changer design has not radically changed in the past two decades [4-6], mainly because the performance has always matched the system requirement. In general, reliability has been the main criterion for a good design to fulfil the relatively slow automatic voltage control characteristics. Hence the conventional mechanical arrangement using oil breaks contactors has been adequate [2, 3],

The more recent innovations in Tap-Changer design have been orientated towards eliminating contact wear and oil pollution to improve reliability and reduce maintenance. Various methods have been employed to reduce the arcing which is accompanied at the contacts, however some contact erosion and oil contamination still take place [2,3].

Conventional transformer Tap-Changers inserts resistance or reactance during the switching operation, and the three or four transition stages often required many cycles of the supply frequency. Solid-state Tap-Changers eliminate the need for switching resistors or reactors and operate in less than one cycle eliminate the maintenance and minimize the arcing associated with switching transition [6-8].

There are several conditions which are very important for the design of the Tap-Changers [1,2]:

$$
\begin{aligned}
& \text { 1- Transformer rating. } \\
& \text { 2- Number of taps. } \\
& \text { 3- Tap voltage. }
\end{aligned}
$$

Furthermore, current should not be interrupted during the operation of the on load tap changer.

\section{Tapping Winding Arrangement}

To select the tapping range from the tap changer few comment arrangements are used. Usually the leads from the winding can be taken off to get required range of the tapping [2,3]. Three methods are in common use for providing tapping at neutral end of a high voltage winding.

\subsection{Tapping by Coarse/fine}

Figure 1 shows the general arrangement of the Coarse/fine tapping. As can be seen from the figure a winding extension (coarse part) set on the main winding and controlled by changeover selector. The fine section is set on the main tapping winding. The lead from the fine section is brought out by a special rotary (one for odd and one for even 
tapping).In this arrangement to cover the range for the required tap, the gauged selectors operates in a sequential operation manner and this would make two revolutions, one revolution with the coarse section out of service and the other after the operation of the range over sector.

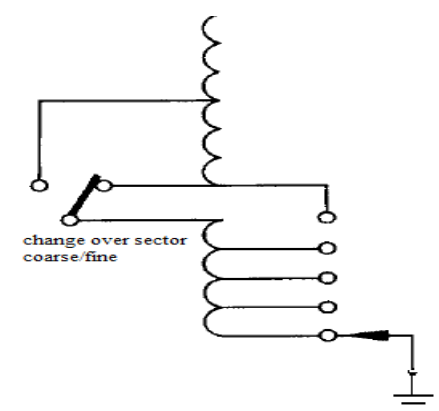

Fig.1. Coarse/ fine tapping arrangement

\subsection{Arrangement by reverse tapping winding}

Figure 2 shows the reverse tapping method. In this arrangement main winding could be boosted or bucked by using a main winding tapping separator. (This can be done by either increasing or decreasing the number of the main winding section in order to change the voltage ratio).

General reverse tapping winding arrangement can be done by tapping ten sections and using two selectors (one for odd and one for even tapping)

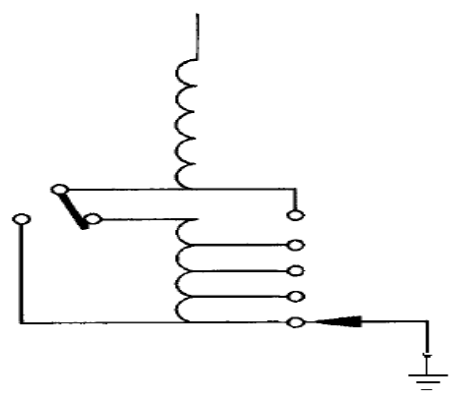

Fig. 2. Reverse tapping winding

\subsection{Linear arrangement}

Figure 3 shows a linear tapping arrangement, it acts like a linear potentiometer which changes the output voltage linearly with the number of tapping. Two type selectors are used for even and odd taps. This arrangement has the advantage of the mechanical design simplicity.

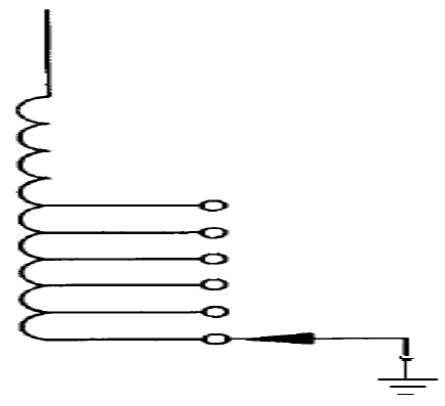

Fig. 3. Linear tapping winding

\section{Thyristors Tap Changer}

To illustrate the principle of operation consider a single phase, using two antiparallel thyristors for each tap as shown in Figure 4 The sequence of events for switching up or down when the load current and voltage are in phase or out of phase is presented below. Switching up will be achieved when operation of thyristors TH3 and TH4 (normal operation) are transferred to thyristors $\mathrm{TH} 1$ and $\mathrm{TH} 2$, the voltage will increase from VB to VA. Switching down will be achieved when thyristors TH5 and TH6 are turned-on and the other thyristors are turned-off, the voltage will decrease to VC. In practice many operational requirements have to be considered and appropriate strategies developed[7-9].

1-At least one pair of inverse parallel connected thyristors must be gated prior to transformer energization.

2- Transient on the load current durring transformer energization or a sudden changing of load.

3- A sudden change of load current in either magnitude and/or phase immediately prior to a tap change instruction.

4- Transformer currents on over load

5- Systems faults. [10-12]

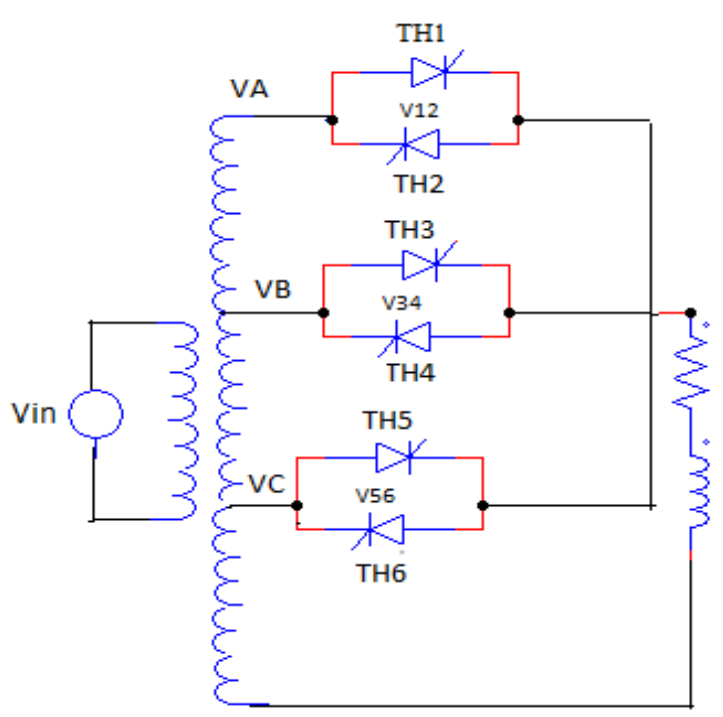

Fig. 4. Thyristors tap changer

First consider the load as a purely resistive (voltage and current are in phase)and the circuit is initialized by gating thyristors $\mathrm{TH} 3$ and $\mathrm{TH} 4$. Then the load current is:

$\mathrm{i}_{\mathrm{L}}=\frac{\mathrm{VB}}{\mathrm{R}} \sin (\omega \mathrm{t})$

To switch up, let TH1 be turned on at any time over a positive half cycle. Then:

Kirchoff's voltage law for the circuit mesh including TH1 and TH2 gives:

$\mathrm{V} 12-\mathrm{V} 34-\mathrm{VAB}=0$

But $\mathrm{V} 12=0$, therefore:

$\mathrm{V} 34=-\mathrm{VAB}$ 
TH3 has a reverse voltage which is ready to commutate. When $\mathrm{TH} 2$ is turned-on to conduct over the negative half cycle, again $\mathrm{V} 12=0$, for the circuit mesh including $\mathrm{TH} 2$ and TH4 equation (2) and(3) apply. Because VAB $<0$, and therefore V34 > 0, TH4 will commutate and the upward transition will take place.

For switching down, let again TH3 and TH4 be turned on throughout alternate half cycles. Let TH5 be turned on at any time over a positive half cycle, therefore:

Kirchoff's voltage law for the circuit mesh including TH5 and TH6 gives:

$\mathrm{V} 34-\mathrm{V} 56-\mathrm{VBC}=0$

But V56 =0, therefore:

$\mathrm{V} 34=\mathrm{VBC}$

Note that: V12, V34 and V56 are the voltage drop across the devices for each tap.

Thus, TH3 will still has a forward voltage and does not turn-off, a short circuit will occur which may damage the system. To avoid the short circuit, TH5 and TH6 must be gated at the instant when the voltage on tap B and tap C is about to change their polarities. Furthermore another important factor is the device turn-off time which affects the phenomena of the switching. If $\mathrm{TH} 3$ has not yet deionized completely, it can be forced into conduction if a forward voltage exists across it. If the device can get its forward blocking capability as soon as the current ceases, switching down transition is accomplished by transferring the conduction from TH3 to TH6 or TH4 to TH5.

The operation with inductive load is not as the same as the resistive load, the current keeps flowing through the device when a reverse voltage exists across it. There is a limit time to switch up or down as illustrated below.

Let TH3 and TH4 again be turned-on through alternate half cycles, then the load current is:

$\mathrm{i}_{\mathrm{L}}=\frac{\mathrm{VB}}{\mathrm{Z}} \sin (\omega \mathrm{t}-\phi)$

$\mathrm{z}=\sqrt{\mathrm{R}^{2}+(\omega \mathrm{L})^{2}}$

$\phi=\tan ^{-1} \frac{\omega \mathrm{L}}{\mathrm{R}}$

Now let TH1 be turned on at time less than the impedance angle $\varphi$ because TH4 is still conducting (the load current still negative) then V34 $=0$, therefore the upper half of the transformer secondary winding will be short circuited through TH1 and TH4. TH1 may not be turned-on until the load current becomes positive, similarly $\mathrm{TH} 2$ may not be turned-on until the load current becomes negative. Thus an upward transition can only takes place when the voltage and current have the same polarities. Downward transition can only take place when the voltage and the current have opposite polarities.

\section{GTOs/Thyristors Tap-Changer}

The use of two antiparallel thyristors as an element switch for a Tap-Changer associated with certain problems as mentioned before mainly with switch down transition. To tackle these problems a GTO with antiparallel thyristor for each tap could be used. This allows switching up or down could be happen at any time when the current flows through the GTO. The block diagram for the circuit configuration is shown in Figure 5, the GTO conducts over a positive half cycle and the thyristor over a negative half cycle. Under normal operation GTO2 and TH2 are gated, for switching up transition let GTO1 be turned on by injecting a positive current into its gate and let GTO2 be turned-off by applying a negative voltage between its gate and cathode. At the same time the driving pulses will be removed from the gate of TH2 and transferred to the gate of $\mathrm{TH} 1$, this will have a reverse voltage which will turned it off. For switching down, first GTO3 will turn-on and GTO2 will turn-off, TH3 will conduct over the negative half cycle while $\mathrm{TH} 2$ will have a reverse voltage which turned it off.

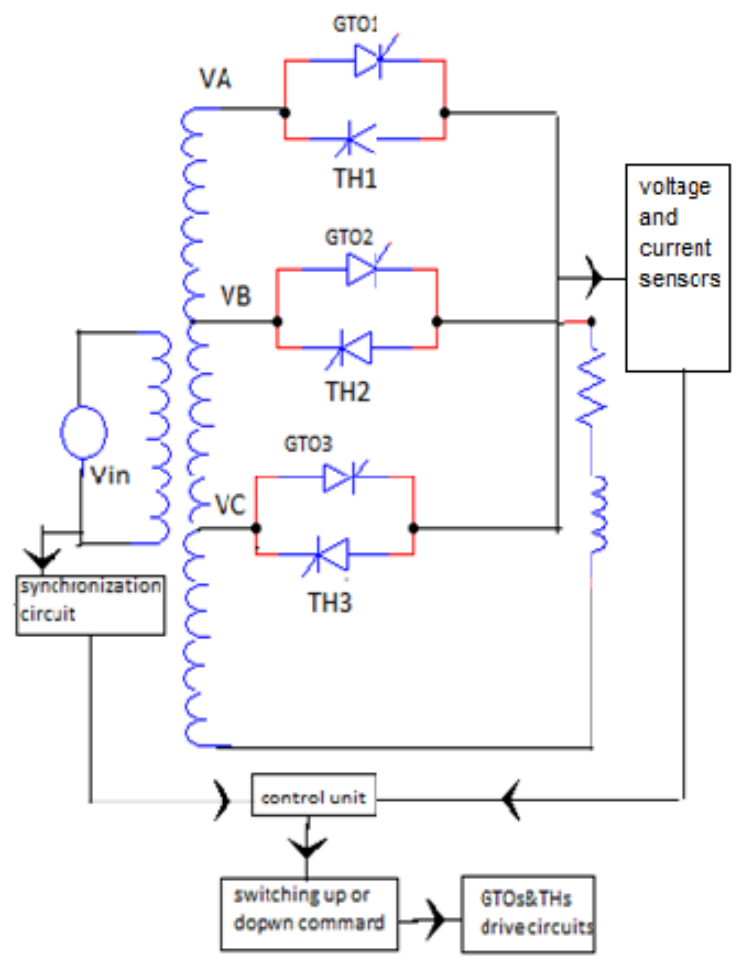

Fig. 5. GTOs/ Thyristors tap changer

A control circuit is designed to ensure that only one tap operates at a time, switching up or down depends on the level of the output voltage and current. These are sensed by voltage and current transformer and feed back to the control circuit via a switching logic circuit which in turn selects the right pulses for the required tap.

Since the three GTOs have the same common point for their cathodes, a single unit power supply is enough for their driving circuits. Referring to Figure 5 witching up or down could be achieved by two different way:

1 - If the input voltage changes, the voltage and current sensors produce signals which are the necessary condition for switching transition. This type of control is very useful, the load voltage could be set to a fixed value. Any variation in 
the input voltage causes the switching logic circuit to choose the right tap to keep the output constant, when the output voltage increases, the normal operation is overridden and a different mode of operation takes place. A voltage transformer is used to sense the output voltage, when this voltage is higher or lower than a reference voltage . the controller automatically choose the switching condition and upward or down ward command will be achieved.

2- Manual switching by choosing the required tap in the transformer. For switching up or down three position slide switches. When the selected switch is closed, its output connects to the control circuit unit which in turn choose the required tap. This type of control is very useful at low voltage for an induction motor starting and can be switched to a rated voltage by a touch of a switch.

\section{Simulation and Experimental Results}

A single-phase GTO / thyristor switched Tap-Changer as shown in Figure 5, was simulated designed and built.

The circuit was designed with power flows in one direction and for switching up or down one step at a time $(45 \mathrm{~V})$, the primary of the transformer is connected to $210 \mathrm{~V}$ RMS input voltage, the. The secondary of the transformer is tapped from 0 to $115 \%$ of the primary voltage. Referring to Figure 5, tap (B) is the normal operation which connected to a point gives $100 \%$ the input, tap (A) is the upward transition which connected to a point gives $115 \%$ the input voltage, and finally tap (C) downward transition is connected to a point which gives $85 \%$ of the input voltage. The circuit was tested under various load conditions, Simulation results for the waveforms of the load voltages and currents for resistive and inductive loads are shown in Figures 6 to 9. Experimental results are shown in figures 10 to 15 .

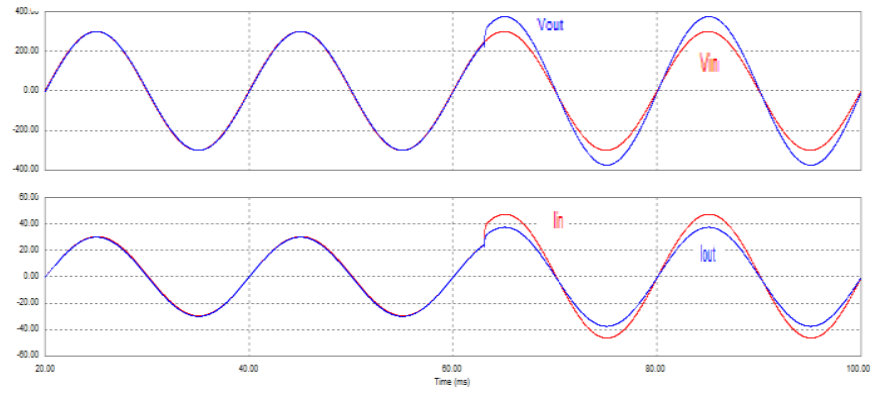

Fig. 6. Switching up simulation, for input and output voltage and current for resistive load

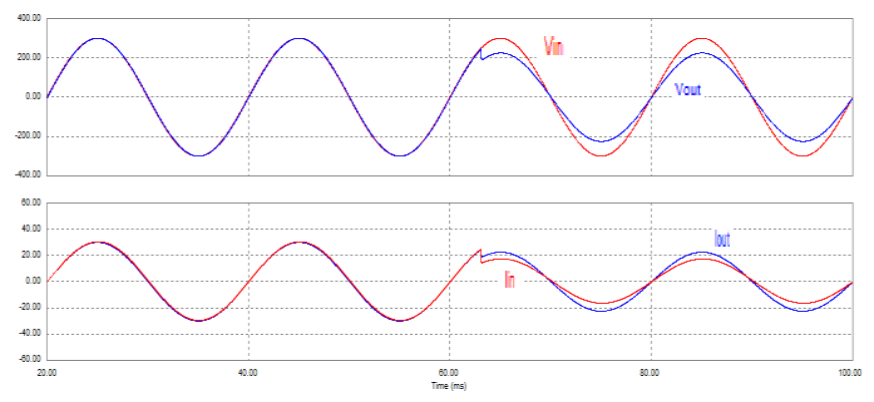

Fig. 7. Switching down simulation, for input and output voltage and current for resistive load

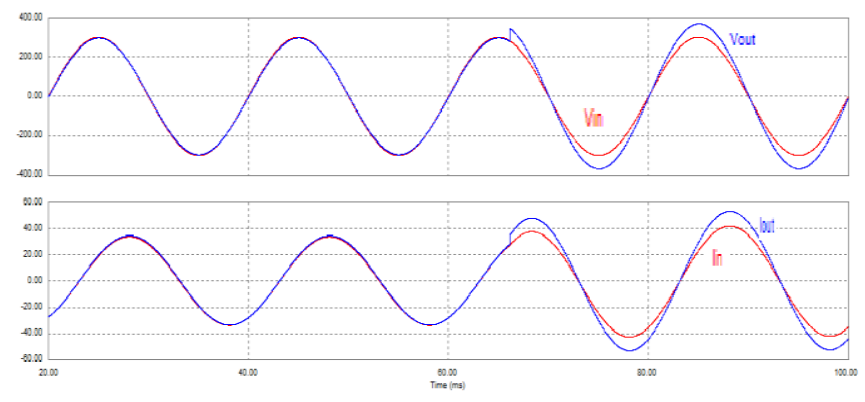

Fig. 8. Switching up simulation, for input and output voltage and current for inductive load

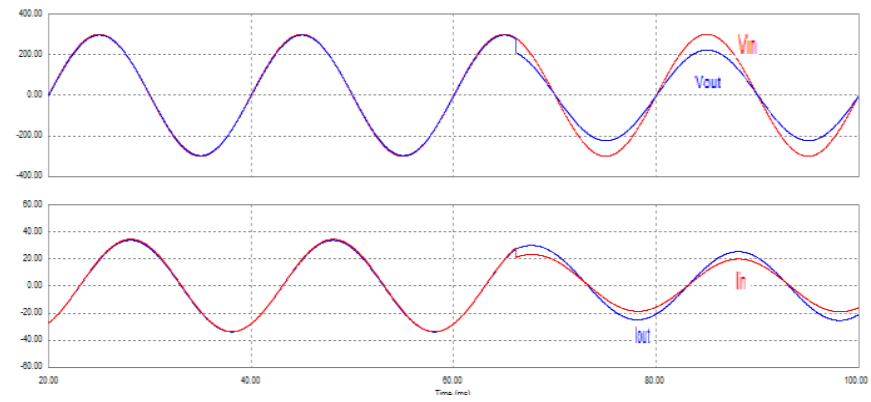

Fig. 9. Switching down simulation, for input and output voltage and current for inductive load

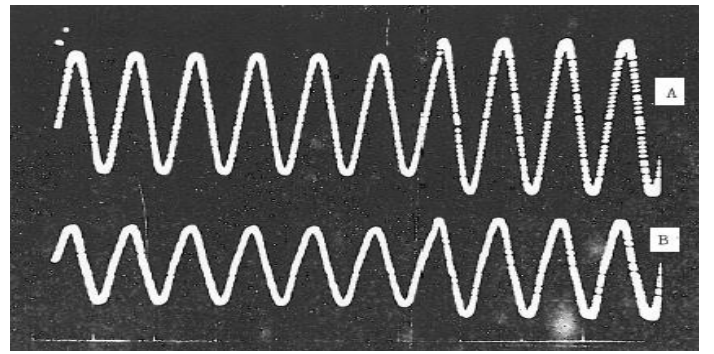

Fig. 10. Load voltage (A) and current (B) for resistive load (switching up)

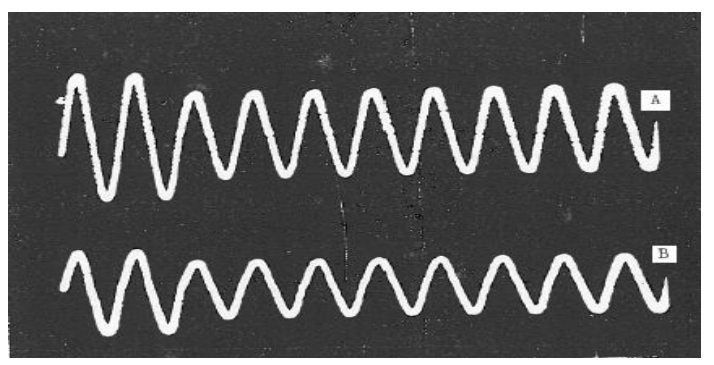

Fig. 11. Load voltage (A) and current (B) for resistive load (switching down)

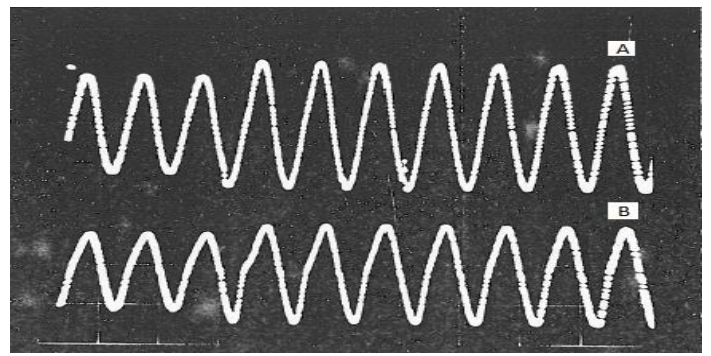

Fig. 12. Load voltage (A) and current (B) for inductive load (switching up) 


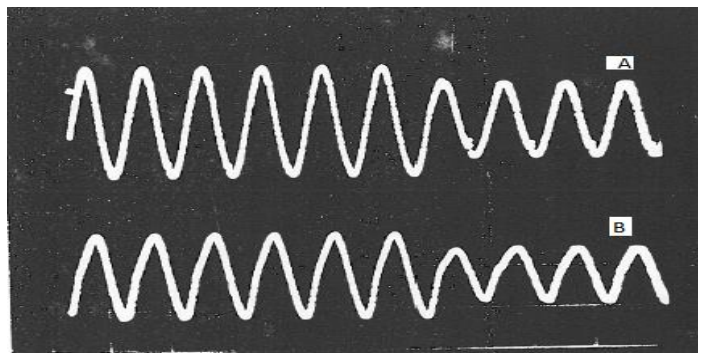

Fig. 13. Load voltage (A) and current (B) for inductive load (switching down)

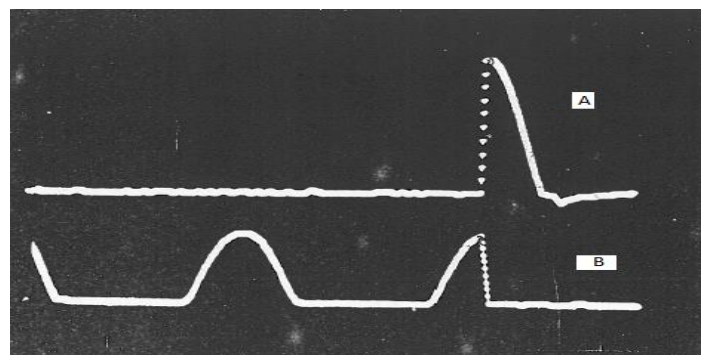

Fig. 14. GTO currents with resistive load (switching up) A- Current through GTO1. B- Current through GTO2

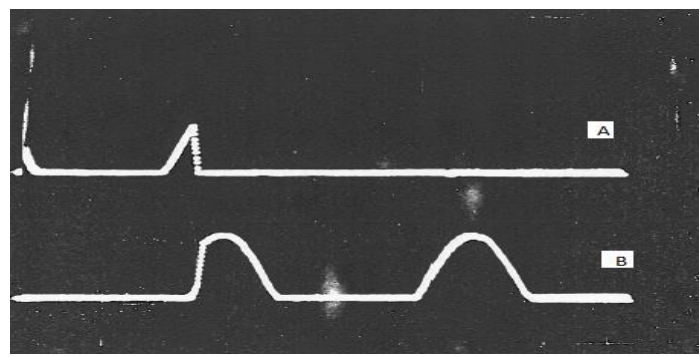

Fig. 15. GTO currents with resistive load (switching down) A- Current through GTO2, B- Current through GTO

\section{Conclusion}

The paper describes the operation of both GTO and thyristor tap changers. For thyristors tap changer it was illustrated that, switching up should be done when voltage and current has the same direction and switching up when they are in opposite direction otherwise a short circuit could occur and damage the system. For GTO tape changer switching up or down could be occurred at any time when the GTO is conducting.

The circuit was responding satisfactorily to the switching transition and this took place with a very low voltage transient across the devices. It is possible to have multi-tap transformers with power flows in either direction or switching up or down to any required voltage within the transformer rating. A microprocessor control could be engaged for deciding the switching and could be used in the electrical distribution networks which would replace the conventional on load Tap-Changes which are extremely expensive and require frequent maintenance.
PSIM software tools used to simulate the proposed tap changer, this verified the performance of the controller of the tap changer. Experimental results showed the system behaviour against any change of the input voltage.

\section{References}

[1] R. Feinberg, "Modern Power Transformer Practice", Macmillan Press Ltd, 1979.

[2] D. O'kelly and G. Musgrave, “An Appraisal of Transformer Tap-Changing Technique”, IEEE Conference Publication, no. 137, pp. 105-109, Jan. 1973.

[3] A.F. Plessis, "Microprocessor Based Power Transformer Voltage Control Scheme", IFAC Symp, Pretoria, South Africa, pp. 165-172, September 1980.

[4] H. Jiang, R. Shuttleworth, B.A.T. Al Zahawi, X. Tian, and A. Power, "Fast response GTO assisted novel tapchanger”, IEEE Trans. Power Del., vol. 16, no. 1, pp. 111-115, Jan. 2001.

[5] R. Shuttleworth, X. Tian, C. Fan, and A. Power, "New tap changing scheme", Inst. Elect. Eng., Electic Power Applications, vol. 143, no. 1, pp. 108-112, Jan. 1996.

[6] J. Harlow, "Discussion of Fast response GTO assisted novel tap changer", IEEE Trans. Power Del., vol. 16, no. 4, pp. 826-827, Oct. 2001.

[7] G.H. Cooke and K.T. Williams, "Thyristor assisted onload tap-changers for transformers", Power Electronics and Variable-Speed Drives, pp. 127-131, Jul. 1990.

[8] G. H. Cooke and K. T. Williams "New thyristor assisted diverter switch for on-load transformer tap-changers", Inst. Elect. Eng., Electric Power Applications, vol.139, no. 6, pp. 507-511, Nov. 1992.

[9] T. Larsson, R. Innanen, and G. Norstrom, "Static electronic tap-changer for electric machines and voltage control", IEEE Fast Phase and Drives Conf., May 1997, pp. TC3/4.1-TC3/4.3.

[10] V. Sanchez, R. Echavarria, M. Cotoragea and A. Claudio, "Design and implementation of a fast on-load tap-changing regulator using soft-switching commutation techniques", PESC, pp. 488-493, Jun. 2000 .

[11] R. Echavarria, V. Sanchez, M. Ponce, M. Cotorogea, and A. Claudio, "Analysis and design of a quasiresonant fast on-load tap changing regulator", J. Circuit, Syst, Comput., vol. 13, no. 4, Aug. 2004.

[12] R. Echavarria, V. Sanchez, M. Ponce, A. Claudio and M. Cotorogea, "Parametric analysis of a quasiresonant fast on-load tap-changing regulator”, PESC, 2002, pp. 1809-1814. 